\title{
ENHANCED MODEL FOR PRECISE POINT POSITIONING WITH SINGLE AND DUAL FREQUENCY GPS/GALILEO OBSERVABLES
}

\author{
A. Afifi, A. El-Rabbany
}

Department of Civil Engineering, Ryerson University, Toronto, Ontario, Canada - (akram.afifi, rabbany@ ryerson.ca)

\section{Technical Commission II}

KEY WORDS: GNSS, PPP, GPS, Galileo, Single frequency, Dual frequency

\begin{abstract}
:
This paper introduces a newly developed model for both single and dual-frequency precise point positioning (PPP), which combines GPS and Galileo observables. As is well known, a drawback of a single GNSS system is the availability of sufficient number of visible satellites in urban areas. Combining GPS and Galileo systems offers more visible satellites to users, which is expected to enhance the satellite geometry and the overall positioning solution. However, combining GPS and Galileo observables introduces additional biases which require rigorous modelling, including the GPS to Galileo time offset (GGTO) and the inter-system bias. This research introduces a new ionosphere-free linear combination model for GPS/Galileo PPP, which accounts for the additional errors and biases. An additional unknown is introduced in the least-squares estimation model to account for the additional biases of the GPS/Galileo PPP solution. It is shown that a sub-decimeter level positioning accuracy and $20 \%$ reduction in the solution convergence time can be achieved with the newly developed GPS/Galileo PPP model.
\end{abstract}

\section{INTRODUCTION}

Precise point positioning (PPP) technique allows a user with a standalone single and dual-frequency global navigation satellite system (GNSS) receiver to determine his or her position at the decimeter level accuracy. The accuracy of PPP depends on the ability to mitigate all errors and biases, which can be achieved through modeling, estimation, and combination of the GNSS observables. PPP relies essentially on the availability and use of precise satellite products, namely orbital and clock corrections. At present, a number of organizations such as the International GNSS Service (IGS) and the Cooperative Network for GIOVE Observations (CONGO) network provide the user with such precise products.

A drawback of a single GNSS system such as GPS is the availability of sufficient number of visible satellites in urban areas. With the addition of Galileo satellites, a PPP solution based on the combined GPS/Galileo measurements becomes more feasible. Combining the two satellite constellations offers more visible satellites to users, which in turn enhances the satellite geometry and is expected to improve the overall positioning solution (Hofmann-Wellenhof et al., 2008). Combining GPS and Galileo, however, comes at the expense of introducing additional biases to the observations mathematical models. These include the GPS to Galileo time offset, and Galileo satellite hardware delay. Recently, the European Space Agency (ESA) estimated the GPS to Galileo time offset (GGTO), which was found to be approximately 50ns, or equivalently $15 \mathrm{~m}$ range error (ESA, 2013). As well, the IGS estimated the code system bias of the GPS/Galileo systems at different stations with different receiver types which has range between -30 and 35 nanoseconds (IGS, 2013).

Afifi and El-Rabbany (2013) showed that combining GPS and Galileo observations in a PPP solution enhances the positioning solution convergence and precision in comparison with GPSonly PPP solution. Their work, however, was limited to singlefrequency data, which is expected to have a relatively longer convergence time for the PPP solution. Melgard at al. (2013) showed that combining multi-constellation in a PPP solution improves the positioning accuracy, especially when the system biases are calibrated. As well, Odijk and Teunissen (2013) showed that prior correction of the differential GPS/Galileo (GIOVE) inter-system biases significantly increases the success rate of instantaneous ambiguity resolution for short baselines. Likewise, Paziewski and Wielgosz (2013) showed that combining GPS/Galileo observables in a double-differenced carrier-phase and pseudorange technique improves the success rate of instantaneous ambiguity resolution in comparison with GPS-only solution. Unfortunately, however, their work was limited to differential positioning techniques.

This paper develops a GPS/Galileo PPP model, which rigorously accounts for the additional combination biases, namely the GPS to Galileo time offset, and Galileo satellite hardware delay. These additional biases are lumped and considered as a new unknown parameter, commonly known as inter-system bias, in the PPP mathematical model. The GPS hardware delay is lumped to the receiver clock error in both GPS-only and GPS/Galileo PPP models. Galileo signals E1/E5b and E1/E5a are combined with the GPS L1/L2 signals in a dualfrequency ionosphere-free linear combinations, respectively. In addition, GPS L1 is combined with Galileo L1 signal in a single-frequency PPP analysis. Sequential least-squares estimation technique is used to get the best estimates for the inter-systems bias parameter. The positioning results of the newly developed GPS/Galileo PPP model showed a subdecimeter accuracy level and $25 \%$ convergence time improvement in comparison with the GPS-only PPP results.

\section{GPS AND GALILEO COMBINATION MODELS}

Generally, the accuracy of PPP depends on the ability to mitigate all errors and biases. GNSS pseudorange and carrierphase measurements are both affected by several types of random and systematic errors. These errors may be classified as those originating at the satellites, those originating at the receiver, and those that are due to signal propagation through the atmospheric layers (El-Rabbany, 2006). GNSS errors attributed to the satellites include satellite clock errors, orbital errors, satellite hardware delay, satellite antenna phase centre variation, and satellite initial phase bias. Errors attributed to signal propagation include the delays of the GNSS signal as it 
passes through the ionospheric and tropospheric layers. Errors attributed to receiver/antenna configuration include, among others, the receiver clock errors, multipath error, receiver noise, receiver hardware delay, receiver initial phase bias, and receiver antenna phase center variations.

In addition to the above errors and biases, combining GPS and Galileo observation in a PPP model introduces additional errors such as GGTO due to the fact that each system uses a different time frame. GPS system uses the GPS time system, which is referenced to coordinated universal time (UTC) as maintained by the US Naval Observatory (USNO). On the other hand, Galileo satellite system uses the Galileo system time (GST), which is a continuous atomic time scale with a nominal constant offset with respect to the international atomic time (TAI) (Hofmann-Wellenhof et al., 2008). Moreover, GPS and Galileo use different reference frames, which should be considered in the combined PPP solution. The mathematical models of GPS and Galileo observables, code and carrier phase, can be written respectively as:

$$
\begin{gathered}
\mathrm{P}_{\mathrm{G}}=\rho_{G}\left(t_{G},(t-\tau)_{G}\right)+\mathrm{c}\left[\mathrm{dt}_{\mathrm{r}}\left(t_{G}\right)-\mathrm{dt}^{\mathrm{s}}(t-\tau)_{G}\right]+T_{G} \\
+I_{G}+c\left[d_{r}\left(t_{G}\right)+d^{s}(t-\tau)_{G}\right]+d_{m p}+e_{P G} \\
\mathrm{P}_{\mathrm{E}}=\rho_{E}\left(t_{E},(t-\tau)_{E}\right)+\mathrm{c}\left[\mathrm{dt}_{\mathrm{r}}\left(t_{E}\right)-\mathrm{dt}^{\mathrm{s}}(t-\tau)_{E}\right]+T_{E} \\
+I_{E}+c\left[d_{r}\left(t_{E}\right)+d^{s}(t-\tau)_{E}\right]+d_{m p}+e_{P E} \\
\Phi_{\mathrm{G}}=\rho_{G}\left(t_{G},(t-\tau)_{G}\right)+\mathrm{c}\left[\mathrm{dt}_{\mathrm{r}}\left(t_{G}\right)-\mathrm{dt}^{\mathrm{s}}(t-\tau)_{G}\right]+T_{G} \\
\quad-I_{G}+c\left[\delta_{r}\left(t_{G}\right)+\delta^{s}(t-\tau)_{G}\right]+\lambda\left[N_{G}+\phi_{r}\left(t_{0}\right)-\phi^{s}\left(t_{0}\right)\right]+\delta_{m p}+\varepsilon_{\varphi G} \\
\Phi_{\mathrm{E}}=\rho_{E}\left(t_{E},(t-\tau)_{E}\right)+\mathrm{c}\left[\mathrm{dt}_{\mathrm{r}}\left(t_{E}\right)-\mathrm{dt}^{\mathrm{s}}(t-\tau)_{E}\right]+T_{E} \\
-I_{E}+c\left[\delta_{r}\left(t_{E}\right)+\delta^{s}(t-\tau)_{E}\right]+\lambda\left[N_{E}+\phi_{r}\left(t_{0}\right)-\phi^{s}\left(t_{0}\right)\right]+\delta_{m p}+\varepsilon_{\varphi E}
\end{gathered}
$$

where the subscript $G$ refers to the GPS satellite system and the subscript $E$ refers to the Galileo satellite system; $\mathrm{P}_{\mathrm{G}}$ and $\mathrm{P}_{\mathrm{E}}$ are pseudorange for the GPS and Galileo systems, respectively; $\Phi_{\mathrm{G}}$ and $\Phi_{\mathrm{E}}$ are the carrier phase measurements of the GPS and Galileo systems, respectively; $\mathrm{dt}_{\mathrm{r}}(\mathrm{t}), \mathrm{dt}^{\mathrm{s}}(\mathrm{t}-\tau)$ are the clock error for receiver at reception time $t$ and satellite at transmitting time $t-\tau$, respectively; $d_{r}(t), d^{s}(t-\tau)$ are frequency dependent code hardware delay for receiver at reception time $t$ and satellite at transmitting time $\mathrm{t}-\tau$, respectively; $\delta_{\mathrm{r}}(\mathrm{t}), \delta^{\mathrm{s}}(\mathrm{t}-\tau)$ are frequencydependent carrier phase hardware delay for receiver at reception time $\mathrm{t}$ and satellite at transmitting time $\mathrm{t}-\tau$, respectively; $T$ is the tropospheric delay; $I$ is ionospheric delay; $d_{m p}$ is code multipath effect; $\delta_{m p}$ is the carrier phase multipath effect; $\lambda$ is the wavelengths of carrier frequencies, respectively; $\Phi_{\mathrm{r}}\left(\mathrm{t}_{0}\right), \Phi^{s}\left(\mathrm{t}_{0}\right)$ are frequency-dependent initial fractional phases in the receiver and satellite channels; $N$ is the integer number of cycles for the carrier phase measurements, respectively; $\mathrm{c}$ is the speed of light in vacuum; and $\rho$ is the true geometric range from receiver at reception time to satellite at transmission time; $\mathrm{e}_{\mathrm{P}}, \varepsilon_{\Phi}$ are the relevant noise and un-modeled errors.

Following Kouba (2009), the basic mathematical model underlying dual-frequency PPP is defined by the ionospherefree linear combination. Inter-system bias is introduced in the GPS/Galileo PPP mathematical model to account for the combined effect of the additional combination biases introduced above, i.e., GGTO, Galileo satellite hardware delay, and the receiver clock difference between both GPS and Galileo systems. Precise orbit and satellite clock corrections of CONGO network are used for both GPS and Galileo satellites (Montenbruck at al., 2009). CONGO's precise satellite clock correction of the GPS satellites includes the effect of the ionosphere-free linear combination satellite hardware delay of GPS L1/L2 signals, which is considered in the GPS/Galileo PPP solution. In addition, the Galileo satellite clock correction produced by CONGO contains the ionosphere-free linear combination satellite hardware delay of the Galileo E1/E5a signals (Montenbruck at al., 2009), which is also considered in the GPS/Galileo PPP solution.

In both of our GPS-only and GPS/Galileo PPP models, the GPS receiver hardware delay is lumped to the receiver clock error. This strategy maintains the consistency of the receiver clock error for both of the GPS and the GPS/Galileo PPP solutions. The difference between the receiver hardware delays of GPS and Galileo signals will be lumped to the inter-system bias as shown in Equations 5 and 6.

$I S B_{\mathrm{a}}=G G T O+\triangle H W D(G P S / \text { Galileo })_{R}$

where $I S B_{a}$ is the inter-systems bias parameter in the PPP mathematical model when using Galileo E1/E5a and GPS L1/L2 signals; $\triangle H W D(G P S / \text { Galileo })_{R}$ is the difference in the receiver hardware delays of the GPS and Galileo signals.

When using Galileo E1/E5b signals, the Galileo satellite hardware delay difference between E1/E5a and E1/E5b signals must be considered and lumped to inter-system bias term as shown in Equation 6.

$I S B_{\mathrm{b}}=G G T O+\Delta H W D(\text { GPS } / \text { Galileo })_{R}+\Delta H W D(\text { Galileo })^{S}$ where $I S B_{b}$ is the inter-systems bias parameter in the PPP mathematical model when using Galileo E1/E5b and GPS

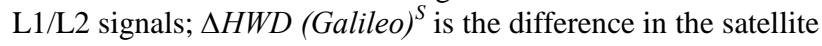
hardware delays of the Galileo E1/E5a and E1/E5b signals. Since the hardware delay and the GGTO are timing errors, they can be combined on one parameter, inter-systems bias, as shown in Equations 7 to 10.

$$
\begin{aligned}
& \mathrm{P}_{3 \mathrm{G}}=\rho+\mathrm{c}\left[\mathrm{dt}_{\mathrm{r}}-\mathrm{dt}_{G}^{s}\right]+T_{G}+e_{P G 3} \\
& \Phi_{3 \mathrm{G}}=\rho+\mathrm{c}\left[\mathrm{dt}_{\mathrm{r}}-\mathrm{dt}_{\mathrm{G}}^{\mathrm{s}}\right]+T_{G}+\lambda \widetilde{N}_{G}+\varepsilon_{\phi G 3} \\
& \mathrm{P}_{3 \mathrm{E}}=\rho+\mathrm{c}\left[\mathrm{dt}_{\mathrm{r}}-\mathrm{dt}_{\mathrm{E}}^{\mathrm{s}}\right]+I S B_{a / b}+T_{E}+e_{P E 3} \\
& \Phi_{3 \mathrm{E}}=\rho+\mathrm{c}\left[\mathrm{dt}_{\mathrm{r}}-\mathrm{dt}_{\mathrm{E}}^{\mathrm{s}}\right]+I S B_{a / b}+T_{E}+\lambda \tilde{N}_{E}+\varepsilon_{\phi E 3}
\end{aligned}
$$

Where $\widetilde{N}$ is the non-integer ambiguity parameter including frequency-dependent initial fractional phases in the receiver and satellite channels; $I S B_{a / b}$ is the inter-systems bias.

\section{SEQUENTIAL LEAST SQUARES ESTIMATION}

Sequential least-squares estimation technique is used to obtain the best estimates of the inter-systems bias parameters. Equations 7 to 10 can be re-arranged for pseudorange and carrier phase observables after applying the satellite clock and the hydrostatic component of the tropospheric zenith path delay tropospheric corrections as follows:

$$
\begin{aligned}
& f_{\mathrm{P}_{\mathrm{G}}}=\rho+\mathrm{cdt} \mathrm{d}_{\mathrm{r}}+z p d_{w G}+e_{P G}-P_{I F_{G}}=0 \\
& f_{\mathrm{P}_{\mathrm{E}}}=\rho+\mathrm{cdt}_{\mathrm{r}}+z p d_{w E}+I S B_{a / b}+e_{P E}-P_{I F_{E}}=0 \\
& f_{\Phi_{\mathrm{G}}}=\rho+\mathrm{cdt} \mathrm{dt}_{\mathrm{r}}+z p d_{w G}+\lambda \tilde{N}_{G}+e_{P G}-\Phi_{I F_{G}}=0 \\
& f_{\Phi_{\mathrm{E}}}=\rho+\mathrm{cdt}+z p d_{w E}+\lambda \tilde{N}_{E}+I S B_{a / b}+e_{P E}-\Phi_{I F_{E}}=0
\end{aligned}
$$

where $z p d_{w}$ is the wet component of the tropospheric zenith path delay. It should be pointed out that other corrections are also applied, including the effects of ocean loading, Earth tide, carrier-phase windup, sagnac, relativity, and satellite and receiver antenna phase-center variations (see Kouba, 2009 for details).

The linearized form of Equations 11 to 14 around the initial parameter $\mathrm{x} 0$ and observables $l$ in matrix form can be written as: 


$$
f(x, l)=A \Delta x-w-r=0
$$

The design matrix $A$ and the vector of corrections to the unknown parameters $\Delta x$ take the following forms:

$$
A=\left[\begin{array}{ccccccccccc}
\frac{x_{0}-X^{1}}{\rho_{0}^{1}} & \frac{y_{0}-Y^{1}}{\rho_{0}^{1}} & \frac{z_{0}-Z^{1}}{\rho_{0}^{1}} & 1 & 1 & 0 & 0 & 0 & 0 & \cdots & 0 \\
\frac{x_{0}-X^{1}}{\rho_{0}^{1}} & \frac{y_{0}-Y^{1}}{\rho_{0}^{1}} & \frac{z_{0}-Z^{1}}{\rho_{0}^{1}} & 1 & 1 & 0 & 1 & 0 & 0 & \cdots & 0 \\
\frac{x_{0}-X^{2^{*}}}{\rho_{0}^{2}} & \frac{y_{0}-Y^{2^{*}}}{\rho_{0}^{2}} & \frac{z_{0}-Z^{2^{*}}}{\rho_{0}^{2}} & 1 & 1 & 1 & 0 & 0 & 0 & \cdots & 0 \\
\frac{x_{0}-X^{2^{*}}}{\rho_{0}^{2}} & \frac{y_{0}-Y^{2^{*}}}{\rho_{0}^{2}} & \frac{z_{0}-Z^{2^{*}}}{\rho_{0}^{2}} & 1 & 1 & 1 & 0 & 1 & 0 & \cdots & 0 \\
\vdots & \vdots & \vdots & \vdots & \vdots & \vdots & \vdots & \vdots & \cdots & \vdots \\
\frac{x_{0}-X^{n}}{\rho_{0}^{n}} & \frac{y_{0}-Y^{n}}{\rho_{0}^{n}} & \frac{z_{0}-Z^{n}}{\rho_{0}^{n}} & 1 & 1 & 0 & 0 & 0 & 0 & \cdots & 0 \\
\frac{x_{0}-X^{n}}{\rho_{0}^{n}} & \frac{y_{0}-Y^{n}}{\rho_{0}^{n}} & \frac{z_{0}-Z^{n}}{\rho_{0}^{n}} & 1 & 1 & 0 & 0 & 0 & 0 & \cdots & 1
\end{array}\right]_{2 \mathrm{n} \times(\mathrm{n}+6)}
$$

where the asterisk '*' refers to Galileo system satellite.

The additional system bias term appears in the Galileo observations equations only. Consequently, the unknowns are the vector of receiver coordinates correction, $\Delta x, \Delta y$, and $\Delta z$, receiver hardware delay, wet component of the tropospheric zenith path delay $z p d_{w}$, the bias term $I S B_{a / b}$, and ambiguities parameters $\widetilde{N}$. The sequential least-square estimation technique can then be written as:

$$
\begin{aligned}
& \Delta x_{i}=\Delta x_{i-1}+M_{i-1}^{-1} A_{i}^{* T}\left[C_{i, i}+A_{i}^{*} M_{i-1}^{-1} A_{i}^{* T}\right]^{-1}\left[w_{i}^{*}-A_{i}^{*} \Delta x_{i-1}\right] \\
& M_{i}^{-1}=M_{i-1}^{-1}-M_{i-1}^{-1} A_{i}^{* T}\left[C_{i, i}+A_{i}^{*} M_{i-1}^{-1} A_{i}^{* T}\right]^{-1} A_{i}^{*} M_{i-1}^{-1} \\
& A_{i}^{*}=A_{i}-C_{i, i-1} C_{i, i-1}^{-1} A_{i-1} \\
& w_{i}^{*}=w_{i}-C_{i, i-1} C_{i, i-1}^{-1} w_{i-1}
\end{aligned}
$$

where $A$ is the design matrix, which includes the partial derivatives of the observation equations with respect to the unknown parameters $X ; \Delta \mathrm{x}$ is the vector of corrections to the unknown parameters $\left(\Delta x=x-x^{0}\right)$; $\mathrm{w}$ is the misclosure vector; $\mathrm{r}$ is the residuals victor; $\mathrm{C}$ is the observations covariance matrix; $M$ is the matrix of the normal equations; $i$ is the epoch index.

\section{RESULTS AND DISCUSSIONS}

Natural Resources Canada's GPSPace PPP software has been modified to handle data from both GPS and Galileo systems, which enables a GPS/Galileo for both single and dual-frequency PPP solution. In addition, the GPSPace PPP software has been modified to handle the new unknown ISB $_{a}$ in case of using GPS L1/L2 and Galileo E1/E5a signals and ISB $_{b}$ in case of using GPS L1/L2 and Galileo E1/E5b signals in the combined PPP model. Hopfield tropospheric correction model has been used along with Vienna mapping function to account for the hydrostatic component of the tropospheric zenith path delay (Hofmann-Wellenhof et al., 2008; Boehm and Schuh, 2004). The IGS global ionospheric maps (GIM) product is used to correct for the ionospheric delay for the single frequency PPP analysis (Schaer et al. 1998).
GPS and Galileo measurements at four well-distributed stations were selected from the IGS tracking network to verify the newly developed GPS/Galileo PPP model. Those stations are occupied by GNSS receivers capable of simultaneously tracking GPS and Galileo measurements. Of them, two are located in Europe, namely DLFT and GOP, while the other two are located in North America, namely UNB and USN. Table 1 show the receiver type of each analysis station.

\begin{tabular}{|c|c|c|c|c|}
\hline & \multicolumn{2}{|c|}{ North America Stations } & \multicolumn{2}{c|}{ European Stations } \\
\hline Station & UNB & USN & DLFT & GOP \\
\hline \multirow{2}{*}{ Receiver } & TRIMBLE & SEPTENTRIO & TRIMBLE & LEICA \\
& NETR9 & polarx4tr pro & NETR9 & GRX1200+GNSS \\
\hline
\end{tabular}

Table 1. Analysis stations information

To study the effect of the receiver hardware delay on the intersystem bias, $I S B_{a / b}$, the analysis stations are picked with different receiver types. Unfortunately, Galileo E5a signal was missing from the observation file at station USN, and therefore was not included in the analysis. As discussed in the Section 2, in order to keep the receiver clock error consistent, the GPS receiver hardware delay is lumped to the receiver clock error in both GPS-only and GPS/Galileo models.

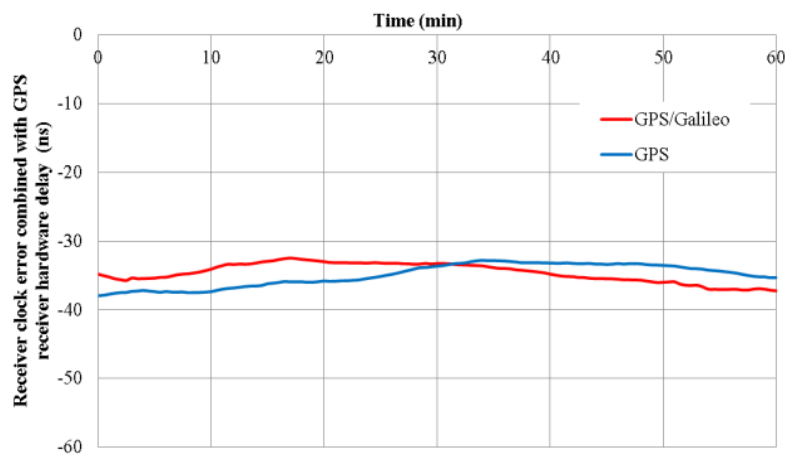

Figure 2. Combined receiver clock error and GPS receiver hardware delay

Figure 2 shows the combined receiver clock error and the GPS receiver hardware delay for both GPS/Galileo and GPS-only PPP solution. As shown in Figure 2, the receiver clock errors of both GPS/Galileo and GPS PPP solutions are consistent as discussed earlier in Section 2.

The results of the single frequency GPS PPP solution and the single-frequency GPS/Galileo PPP solution are obtained using the developed exponential function by Afifi and El-Rabbany (2013). Figures 3 and 4 show the PPP results of the combined GPS/Galileo observations with the sine and exponential functions, respectively.

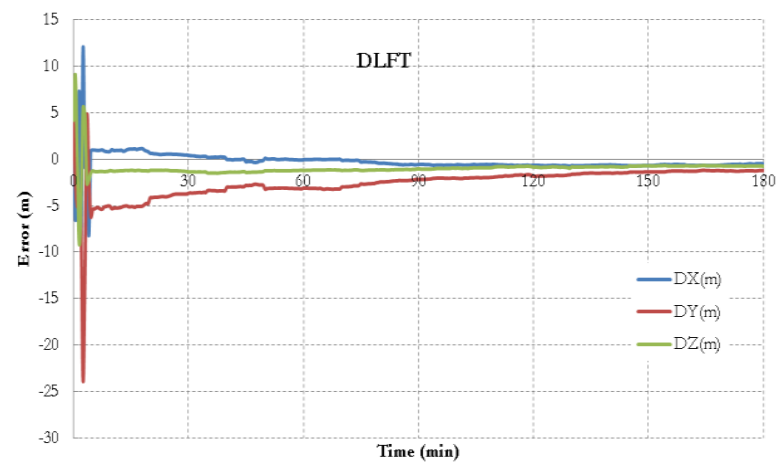


Figure 3. GPS/Galileo PPP results using empirical sine function stochastic model

As can be seen in Figure 3, the results of the GPS/Galileo PPP with the sine function show decimetre-level accuracy; however the solution convergences to this accuracy level after about three hours. Figure 4 shows that, when the exponential function is used, the solution converges to decimetre-level after 30 minutes or less. This is considered significant improvement, especially with single-frequency observations.

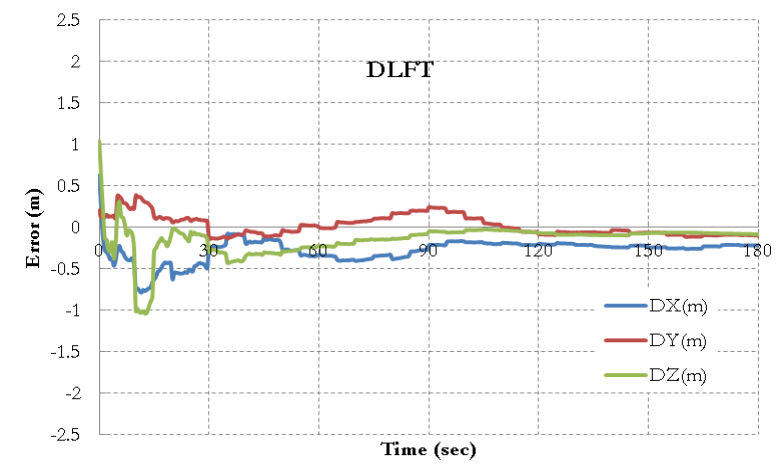

Figure 4. GPS/Galileo PPP results using the newly developed stochastic model

The results of the dual-frequency GPS/Galileo PPP are compared with those of GPS-only PPP solution. Only the positioning results of stations DLFT and UNB are presented in this paper. Similar results were obtained at other stations. As can be seen in Figure 5, the results of dual-frequency GPS PPP show a sub-decimeter accuracy level and a convergence time of about 20 minutes.

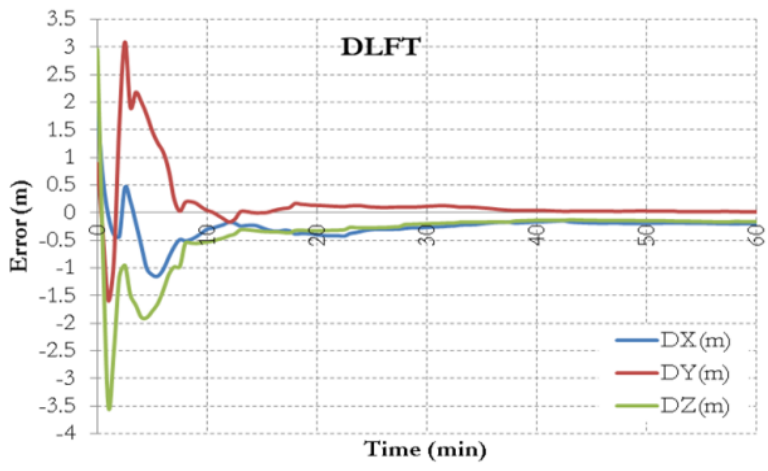

Figure 5. Dual-frequency GPS PPP

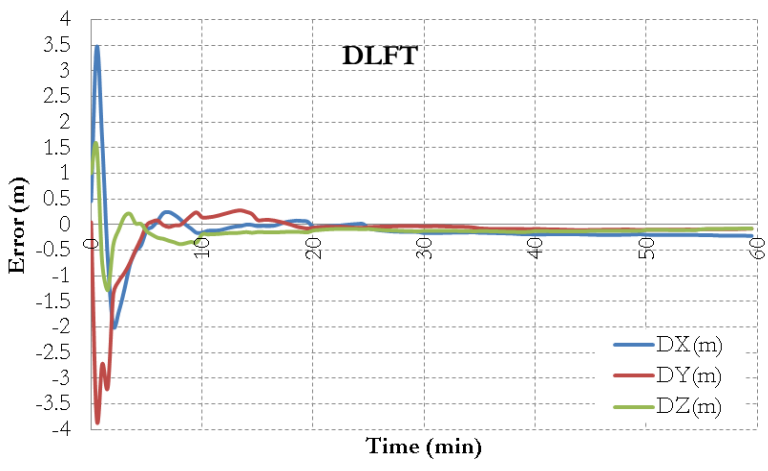

Figure 6. Dual-frequency GPS (L1/L2)/Galileo (E1/E5b) PPP
Figure 6 shows the PPP results for the combined GPS L1/L2 and Galileo E1/E5b signals for both DLFT and UNB stations. The results show a sub-decimeter positioning accuracy and about 15 minutes convergence time, which represents an improvement of about $25 \%$ in comparison with the GPS-only solution.

\section{CONCLUSIONS}

This paper developed a new PPP model using GPS/Galileo observables. The newly developed GPS/Galileo model accounts for the combined effect of GGTO and Galileo satellite hardware delays through the introduction of a new unknown parameter, inter-systems bias or $I S B_{a / b}$, in the PPP mathematical model. It has been shown that $I S B_{a / b}$ is essentially constant over the observation time span with different magnitude ranging between 30 and 60 nanoseconds, depending on the GNSS receiver type. The results of the PPP single-frequency GPS/Galileo show that a sub-decimeter level accuracy is possible in static mode. The single frequency GPS/Galileo results show a significant improvement from three hours to 30 minutes. In addition, It has also been shown that the dualfrequency positioning results of the GPS-only and GPS/Galileo PPP are comparable and are at the sub-decimeter level accuracy. However, the convergence time of the combined GPS/Galileo PPP has improved by about $25 \%$ in comparison with the GPSonly PPP.

\section{ACKNOWLEDGMENTS}

This research was partially supported by the Natural Sciences and Engineering Research Council (NSERC) of Canada, the Government of Ontario, and Ryerson University. The authors would like to thank the International GNSS service (IGS) network and the COoperative Network for GIOVE Observations (CONGO) for providing the satellites precise products.

\section{REFERENCES}

Boehm, J. and H. Schuh (2004). "Vienna mapping functions in VLBI analyses." Geophysical Research Letters 31(1): L01603 01601-01604.

Collins, P., S. Bisnath, et al. (2010). "Undifferenced GPS ambiguity resolution using the decoupled clock model and ambiguity datum fixing." Navigation, Journal of the Institute of Navigation 57(2): 123-135.

El-Rabbany, A. (2006). Introduction to GPS: The Global Positioning System. Artech House Publishing.

Elsobeiey, M. and A. El-Rabbany (2012). "An Improved Model For Precise Point Positioning With Modernized Glogbal Positioning System" (2012). Theses and dissertations. Paper 1324. http://digitalcommons.ryerson.ca/dissertations/1324

European Space Agency (ESA), 2013. Galileo and GPS 'Synchronise Watches': New Time Offset Helps Working Together. Retrieved May 18, 2013, from (http://www.esa.int/Our_Activities/Navigation/Galileo_and_GP S_synchronise_watches_new_time_offset_helps_working_toget her)

International GNSS service (IGS), 2013. Multi-GNSS differential code biases (DCBs), Retrieved December 06, 2013. 
ftp://cddis.gsfc.nasa.gov/pub/gps/products/mgex/dcb/

Hofmann-Wellenhof, B., H. Lichtenegger, and E. Wasle (2008). "GNSS global navigation satellite systems; GPS, Glonass, Galileo \& more". Springer Wien New York. 501pp.

Hopfield, H. S. (1972). Tropospheric refraction effects on satellite range measurements. APL Technical Digest, 11(4), 1119.

Kouba, J. (2009). A guide to using international GNSS service (IGS) products. Available at http://igscb.jpl.nasa.gov/igscb/resource/pubs/UsingIGSProducts Ver21.pdf

Kouba, J. and P. Heroux (2001) "Precise point positioning using IGS orbit and clock products." GPS Solutions, 5(2), PP 12-28.

Melgard, T., Tegedor, J., de Jong, K., Lapucha, D., Lachapelle, G. (2013). "Interchangeable Integration of GPS and Galileo by Using a Common System Clock in PPP." ION GNSS+ September 16-20, 2013, Institute of navigation, Nashville TN, U.S.

Montenbruck, O., Hauschild, A., Hessels, U., Steigenberger, P., Hugentobler, U. (2009). "CONGO first GPS/Giove tracking network for science, research.” GPS World, 20(9), 56-62

Odijk, D. and P. J. G. Teunissen (2013). "Characterization of between-receiver GPS-Galileo inter-system biases and their effect on mixed ambiguity resolution." GPS Solutions 17(4): 521-533.

Paziewski, J. and P. Wielgosz (2013). "Assessment of GPS + Galileo and multi-frequency Galileo single-epoch precise positioning with network corrections." GPS Solutions: 1-9.

Schaer, S., W. Gurtner, J. Feltens. 1998. IONEX: The IONosphere Map EXchange Format Version 1. Proceedings of the IGS AC Workshop, Darmstadt, Germany, 9-11 February. 